\title{
Ovarian Cancer Substantial Risk Factor Analysis by Machine Learning: a Low Incoming Country Perspective
}

\author{
Md. Raihan Ahmed ${ }^{1}$ (D), Hasin Rehana ${ }^{2}$ (D), Sayed Asaduzzaman 3,* (D) \\ 1 Department of Software Engineering, Daffodil International University, Dhaka, Bangladesh; raihan35-1416@ diu.edu.bd, \\ (M.R.A.); \\ 2 Department of Computer Science and Engineering, Daffodil International University, Dhaka, Bangladesh; \\ hasin.cse@diu.edu.bd, (H.R.); \\ 3 Department of Computer Science and Engineering, Rangamati Science and Technology University, Rangamati, \\ Bangladesh; asadcse.rmstu@gmail.com, (S.A.); \\ * Correspondence: asadcse.rmstu@gmail.com; samonna25@gmail.com;
}

Scopus Author ID 57003595300

Received: 2.07.2020; Revised: 25.07.2020; Accepted: 26.07.2020; Published: 28.07.2020

Abstract: In this paper, ovarian cancer data were inspected to figure out the significant risk factors. According to the American Cancer Society, it is the fifth leading cause of death of women. It characterizes that in 2019, 22,530 women will be diagnosed, whereas 22,240 women were diagnosed in 2018, and 13,980 will face death in 2019, but 14,070 women were died because of ovarian cancer. For this research, 521 woman's data were collected from Hospitals of Dhaka with case group 267 and control group 254. A set of a questionnaire of 47 factors that were elicited from various researches used for data collection. Data were examined with different machine learning algorithms like using SVM, logistics regression, random forest, naïve bayes, neural network, $\mathrm{kNN}$, ada boost, $\mathrm{CN} 2$ rule, Decision tree, Quadratic Classifier. These algorithms were compared with each other with different tools and found that Logistics Regression provides the highest accuracy of 0.933 along with the highest CA of 0.848. Data were investigated with ranker algorithms to found out the rankings between factors with the help of feature selection. Significant factors like problems during pregnancy, abortion, cervical cancer history, menopause problems, etc., were found out as significant risk factors of ovarian cancer.

Keywords: overian cancer; data mining; statistics; low incoming country; risk factors.

(C) 2020 by the authors. This article is an open-access article distributed under the terms and conditions of the Creative Commons Attribution (CC BY) license (https://creativecommons.org/licenses/by/4.0/).

\section{Introduction}

In female reproductive organs, ovaries or the fallopian tubes were disturbed, and cells appeared beyond necessity is ovarian cancer. Ovarian cancer consists of the top stage of tumor heterogeneity along with complexity. Epithelial ovarian cancer forms mostly of ovarian cancers and is the common cause of death of women by cancer [1,2]. BRAC1 and BRAC 2 are two common genes that cause ovarian cancer, whereas family history impacts on cancer [3]. In [4], research on ovarian cancer takes place where the analysis shows the significance of the long-term raised chance for mucinous ovarian cancer and benign ovarian tumors. Inflammatory damage to the fallopian tube induced by $C$. trachomatis salpingitis and could potentially create ovarian cancer $C$. trachomatis, a sexually transmitted infection may cause ovarian cancer [5, $6]$.

A number of research has been taken place to preclude ovarian cancer. Plant oil $\beta$ Caryophyllene (BCP) perform cell cycle arrest and apoptosis in ovarian cancer. So it can be an anti-cancer agent [7]. Clinical trials have been implemented to cure ovarian cancer [8]. 
Infertility and Ovarian cancer can be interrelated with breast cancer. Early-stage of breast cancer shows few symptoms that tend to serious causes of ovarian cancer [9]. In [10] shows, The late diagnosis of ovarian cancer and related problem cause a huge impact on women. The human immune system by blockade of the PD-1/PD-L1 axis has evidenced a positively clinical trial on ovarian cancer. A cross-resistance based model which has been proposed in [11] shows that cancer cells may be bounded by HLA chemotherapy- resistance. The risk of Ovarian cancer can be decreased if it can be screened at an early stage [12]. Ovarian tumors result in ovarian cancer illustrated in [13], which shows population-based survey research. Advanced age, menopause, weight loss, large cyst diameter are some of the important factors of ovarian cancer illustrate from case-control research [14]. Hormonal facts cause ovarian cancer. A combination of 2D light scattering anisotropy cytometry with machine learning-based, a labelfree cytometric technique has been developed to classify single cells from ovarian cancer [15]. Machine learning algorithm Support Vector Machine (SVM) results of both breast cancer and ovarian cancer depict the model of performance for classification tasks [16]. Different machine learning approaches have been demonstrated to predict the risk factors of ovarian cancer. Gene expression patterns of ovarian cancer can be detected by machine learning and statistical approaches $[17,18]$. The merge of Convolution Neural Network and Relif that are two models of machine learning has been used to classify and predict the risk factors of ovarian cancer [19].

Hormonal imbalance can cause ovarian cancer [20]. Our research on the ovarian cancer dataset takes place by machine learning approach as well as statistical approaches. The research shows that significant risk factors with their level of significance have been observed and depicted. The methodology part illustrates the initial data collection and data cleaning process, whereas the results and discussion section shows the detailed output by which we extract the risk factors of ovarian cancer.

\section{Materials and Methods}

The detailed materials and methods have been described as follows

\subsection{Data Collection and pre-processing.}

The dataset was collected from different hospitals in Dhaka, Bangladesh. A questionnaire was prepared from previous studies, and it was used for data collection. There were 47 factors in the questionnaire, and each and every individual was asked for data, and the meeting was coordinated by standard personnel in the hospital. The dataset contains case and control groups for a detailed analysis of the disease. In the dataset complete 47 factors like a problem during pregnancy, a number of children, age, age of husband, affected by Breast cancer, having an infection in the genital area, affected by cervical cancer, consume of a tentative drug as well as estrogen pill after menopause, abortion and other relevant factors were taken into account for the investigation of deadly cancer.

After data collection, the incompatible, lost, and unsorted data were filtered and cleaned before processing. This phase is easily done by WEKA(a data mining tool) and Orange(a machine learning tool), but sci-kit learn a function for cleaning and training dataset. 


\subsection{Data mining approach.}

Important factors were elicited with the help of WEKA and Orange at the same time sci-kit learn was used for comparing the accuracy of machine learning models. Info gain, gain ratio, gini index, and chi-square test were analyzed with the help of Orange. The ranker algorithm of WEKA was used for feature selection. By these methods, the significance level among the factors is explored on the Dataset. For determining the most significant factors, chisquare, info-gain, gain-ratio, and attributes derived from evaluators and the ranking of Orange were taken into account.

\subsection{Significance formulation.}

Due to the increase in unnecessary epithelial cells in a carcinoma are the main reason for the development of ovarian cancer. From the analysis, a probability range and useful box plot for different factors influencing ovarian cancer can be derived. To determine an individual $\mathrm{s}$ affected or not, the probability range and the box plot can be used. Previously disease risk prediction and detailed analysis were made for developing a model that can accurately predict the risk of Diabetes by S. Asaduzzaman et al. [21].

\section{Results and Discussion}

SVM, Random Forest, Logistic regression, Adaboost, Neive Bias are some models of machine learning. These models or algorithms can predict the accuracy of the dataset on data models. Here to predict the accuracy along with precision, support, CA has been analyzed. The highest accuracy of 0.933 with an orange tool for the logistic regression model has been discovered and shown in Table 1.

Table 1. Comparison among the classifiers using machine learning algorithms.

\begin{tabular}{|c|c|c|c|c|c|c|c|c|}
\hline \multirow[b]{2}{*}{ Model } & & & & & & & & \\
\hline & Tool & AUC & CA & F1 & Precision & Recall & Specificity & Support \\
\hline \multirow{2}{*}{ SVM } & Orange & 0.921 & 0.835 & 0.835 & 0.841 & 0.835 & 0.838 & - \\
\hline & Sklearn & 0.761 & - & 0.76 & 0.77 & 0.76 & - & 126 \\
\hline \multirow{2}{*}{$\begin{array}{l}\text { Random } \\
\text { Forest }\end{array}$} & Orange & 0.926 & 0.843 & 0.843 & 0.843 & 0.843 & 0.843 & - \\
\hline & Sklearn & 0.778 & - & 0.78 & 0.79 & 0.78 & - & 126 \\
\hline \multirow{2}{*}{$\begin{array}{l}\text { Logistic } \\
\text { Regression }\end{array}$} & Orange & 0.933 & 0.848 & 0.848 & 0.848 & 0.848 & 0.848 & - \\
\hline & Sklearn & 0.793 & - & 0.79 & 0.79 & 0.79 & - & 126 \\
\hline \multirow{2}{*}{ AdaBoost } & Orange & 0.900 & 0.827 & 0.827 & 0.827 & 0.827 & 0.827 & - \\
\hline & Sklearn & 0.762 & - & 0.76 & 0.76 & 0.76 & - & 126 \\
\hline \multirow{2}{*}{ Naïve Bayes } & Orange & 0.921 & 0.750 & 0.740 & 0.790 & 0.750 & 0.741 & - \\
\hline & Sklearn & 0.785 & - & 0.78 & 0.79 & 0.78 & - & 126 \\
\hline \multirow{2}{*}{$\begin{array}{l}\text { Neural } \\
\text { Network }\end{array}$} & Orange & 0.912 & 0.814 & 0.814 & 0.814 & 0.814 & 0.813 & - \\
\hline & Sklearn & 0.770 & - & 0.77 & 0.77 & 0.77 & - & 126 \\
\hline \multirow{2}{*}{ kNN } & Orange & 0.917 & 0.839 & 0.839 & 0.839 & 0.839 & 0.839 & - \\
\hline & Sklearn & 0.761 & - & 0.76 & 0.77 & 0.76 & - & 126 \\
\hline \multirow{2}{*}{$\begin{array}{ll}\mathrm{CN} 2 & \text { rule } \\
\text { Inducer } & \\
\end{array}$} & Orange & 0.912 & 0.816 & 0.816 & 0.816 & 0.816 & 0.815 & - \\
\hline & Sklearn & - & - & - & - & - & - & - \\
\hline \multirow{2}{*}{$\begin{array}{l}\text { Decision } \\
\text { Tree }\end{array}$} & Orange & 0.773 & 0.835 & 0.835 & 0.835 & 0.835 & 0.835 & - \\
\hline & Sklearn & 0.754 & - & 0.75 & 0.76 & 0.75 & - & 126 \\
\hline \multirow{2}{*}{$\begin{array}{l}\text { Quadratic } \\
\text { Classifier }\end{array}$} & Orange & - & - & - & - & - & - & - \\
\hline & Sklearn & 0.762 & - & 0.76 & 0.77 & 0.76 & - & 126 \\
\hline
\end{tabular}

Table 2 shows the significant risk factors with their chi-square value. Here problem during pregnancy $\left(\chi^{2}=148.536\right)$ and abortion $\left(\chi^{2}=118.676\right)$ shows the highest chi-square means they are the highest significance. Other factors like Have an infection in Genital Area> Menopose>afffected by cervical cancer are organized in a decreased manner in the Table. The factors with low chi-square value $\left(\chi^{2}\right)$ have been removed for future assessment. Take tentative 
drug and knowledge about ovarian cancer, although it shows high chi-square value it is the overall feedback that we have not fully considered.

Table 2. Significant factors with the help of info gain, gain ratio, gini index and chi-square test.

\begin{tabular}{|c|c|c|c|c|c|}
\hline Attributes & \# & Info. gain & Gain ratio & Gini & $\chi^{2}$ \\
\hline Problem during pregnancy? & 2 & 0.408 & 0.414 & 0.250 & 148.636 \\
\hline Abortion? & 2 & 0.355 & 0.355 & 0.225 & 118.676 \\
\hline Have infection in genital area & 2 & 0.342 & 0.356 & 0.213 & 84.280 \\
\hline Menopose & 3 & 0.270 & 0.181 & 0.153 & 47.347 \\
\hline Affected by cervical cancer? & 2 & 0.264 & 0.308 & 0.160 & 47.090 \\
\hline Affected by breast cancer & 2 & 0.251 & 0.278 & 0.157 & 52.243 \\
\hline Knowledge about ovarian cancer? & 2 & 0.247 & 0.296 & 0.149 & 114.100 \\
\hline Take a tentative drug? & 2 & 0.246 & 0.281 & 0.152 & 111.921 \\
\hline Cloase relative affeted by breast cancer & 2 & 0.241 & 0.287 & 0.146 & 40.581 \\
\hline Berast cancer prior to age 40 & 2 & 0.240 & 0.284 & 0.146 & 41.612 \\
\hline Estrogen pill taking after menopose & 2 & 0.237 & 0.291 & 0.142 & 110.485 \\
\hline Menopause after 50? & 2 & 0.231 & 0.276 & 0.141 & 39.288 \\
\hline Ever had a hysterectomy & 2 & 0.228 & 0.268 & 0.141 & 40.543 \\
\hline Condom/ Diaphram & 2 & 0.221 & 0.274 & 0.134 & 104.769 \\
\hline Any birth control pill? & 2 & 0.221 & 0.232 & 0.143 & 93.780 \\
\hline Use napkin & 2 & 0.220 & 0.263 & 0.135 & 103.446 \\
\hline Any replacement therapy taken & 2 & 0.214 & 0.249 & 0.134 & 100.124 \\
\hline Pregnency after 35 & 2 & 0.212 & 0.245 & 0.133 & 39.975 \\
\hline Pap test? & 2 & 0.211 & 0.246 & 0.132 & 99.114 \\
\hline Childern after 35 & 2 & 0.208 & 0.237 & 0.131 & 40.476 \\
\hline Regular exercise? & 2 & 0.206 & 0.213 & 0.135 & 85.197 \\
\hline Takes hormone after menopose & 2 & 0.206 & 0.251 & 0.127 & 98.322 \\
\hline Diat maintain & 2 & 0.200 & 0.238 & 0.125 & 95.336 \\
\hline Cancer history in the family? & 2 & 0.197 & 0.215 & 0.127 & 43.440 \\
\hline Take adequate fruit & 2 & 0.185 & 0.187 & 0.122 & 72.132 \\
\hline STI? & 2 & 0.171 & 0.200 & 0.110 & 32.078 \\
\hline Food contains high fat? & 2 & 0.113 & 0.151 & 0.071 & 58.026 \\
\hline Age & 3 & 0.107 & 0.148 & 0.060 & 46.190 \\
\hline Menopause end age? & 3 & 0.100 & 0.063 & 0.067 & 7.758 \\
\hline Oral contraception & 2 & 0.099 & 0.163 & 0.059 & 52.634 \\
\hline Education & 3 & 0.070 & 0.058 & 0.047 & 3.586 \\
\hline Age of husband & 4 & 0.069 & 0.081 & 0.042 & 5.796 \\
\hline BMI? & 3 & 0.061 & 0.085 & 0.039 & 6.488 \\
\hline Social class & 3 & 0.055 & 0.045 & 0.037 & 1.028 \\
\hline Height? & 3 & 0.013 & 0.016 & 0.009 & 13.957 \\
\hline Family members & 3 & 0.012 & 0.010 & 0.008 & 0.293 \\
\hline Cancer vaccine taken? & 2 & 0.007 & 0.031 & 0.005 & 4.727 \\
\hline First sex age? & 2 & 0.005 & 0.005 & 0.003 & 1.736 \\
\hline Source of knowledge? & 3 & 0.003 & 0.002 & 0.002 & 0.010 \\
\hline Marital status? & 2 & 0.002 & 0.099 & 0.001 & 0.002 \\
\hline How many children? & 3 & 0.002 & 0.002 & 0.001 & 0.101 \\
\hline Never pregnant? & 2 & 0.001 & 0.005 & 0.001 & 0.701 \\
\hline Smoker? & 2 & 0.001 & 0.004 & 0.000 & 0.441 \\
\hline Obese? & 2 & 0.000 & 0.005 & 0.000 & 0.253 \\
\hline Take alcohol? & 2 & 0.000 & 0.000 & 0.000 & 0.011 \\
\hline
\end{tabular}

Table 3, Table 4, and Table 5 show the ranking output of different algorithms and attribute evaluators. In all cases problem during pregnancy, menopause problem, infection in the genital area, breast cancer history, abortion history shows the highest-ranking value. The values of ranking by the algorithm have been ordered as top-level factors show the highest significance. BMI also affects ovarian cancer; the box plot with age distribution has been shown in Figure 1. Women with BMI than 29 show the highest risk of ovarian cancer. Each factor has been crossmatched in this way.

Table 3. Data table on the significance of factors with the help of feature selection.

Classifier Attribute Eval

A. Condom/ Diaphram
Correlation Attribute Eval

A. Problem during pregnancy?
Gain Ratio Attribute Eval

A. Problem during pregnancy? 


\begin{tabular}{|c|c|c|}
\hline Classifier Attribute Eval & Correlation Attribute Eval & Gain Ratio Attribute Eval \\
\hline B. Affected by breast cancer & B. Abortion? & $\begin{array}{l}\text { B. Have an infection in the genital } \\
\text { area }\end{array}$ \\
\hline C. Family members & C. Have an infection in the genital area & C. Abortion? \\
\hline D. smoker? & D. Affected By cervical cancer? & D. Affected by cervical cancer? \\
\hline E. Affected by cervical cancer? & E. Affected by breast cancer & $\begin{array}{l}\text { E. Knowledge about ovarian } \\
\text { cancer? }\end{array}$ \\
\hline F. Height? & F. Take a tentative drug? & $\begin{array}{l}\text { F. Estrogen pill-taking after } \\
\text { menopause }\end{array}$ \\
\hline G. How many children? & G. Knowledge about ovarian cancer? & $\begin{array}{l}\text { G. Close Relative affected by Breast } \\
\text { Cancer }\end{array}$ \\
\hline H. Ever had a hysterectomy & H. Breast cancer prior to age 40 & H. Breast cancer prior to age 40 \\
\hline $\begin{array}{l}\text { I. Close relative affected by breast } \\
\text { cancer }\end{array}$ & I. Close relative affected by breast cancer & I. Take a tentative drug? \\
\hline J. Breast cancer prior to age 40 & J.Any birth control pill? & J. Affected by breast cancer \\
\hline K. Menopause after 50 ? & K. Estrogen pill-taking after menopause & K. Menopause after 50? \\
\hline L. Any replacement therapy taken & L. Menopause after 50 ? & L. Condom/ Diaphram \\
\hline M. pap test? & M. Ever had a hysterectomy & M. Ever had a hysterectomy \\
\hline $\begin{array}{l}\text { N. Estrogen pill taking after } \\
\text { menopause }\end{array}$ & N. Use napkin & N. Use napkin \\
\hline O. Menopause & O. Regular exercise? & O. Takes hormone after menopause \\
\hline P. Pregnancy after 35 & P. Any replacement therapy has taken & $\begin{array}{l}\text { P. Any replacement therapy has } \\
\text { taken }\end{array}$ \\
\hline Q. Children after 35 & Q. Condom/ Diaphram & Q. pap test? \\
\hline R. Oral Contraception & R. Pregnency after 35 & R. Pregnency after 35 \\
\hline S. Takes hormone after menopause & S. Pap test? & S. Diet maintain \\
\hline T. Social class & T. Childern after 35 & T. Childern after 35 \\
\hline U. Use napkin & U. Cancer history in the family? & U. Any birth control pill? \\
\hline V. Diet maintain & V. Takes hormone after menopause & V. Cancer history in the family? \\
\hline W. STI? & W. Diat maintain & W. Regular exercise? \\
\hline X. Cancer history in the family? & X. Take adequate fruit & X. STI? \\
\hline Y. Education & Y.STI? & Y. Take adequate fruit \\
\hline Z. Obese? & Z. Food contains high fat? & Z. Menopause \\
\hline AA. BMI? & AA. Oral contraception & AA. Oral contraception \\
\hline AB. Take a tentative drug? & AB. Menopause & AB. Food contains high fat? \\
\hline AC. Take alcohol? & AC. BMI? & AC. Age \\
\hline $\begin{array}{l}\text { AD. Knowledge about ovarian } \\
\text { cancer? }\end{array}$ & AD. Education & AD. Marital status? \\
\hline AE. Age of husband & AE. Menopause end age? & AE. BMI? \\
\hline AF. Source of knowledge? & AF. Age of husband & AF. Age of husband \\
\hline $\begin{array}{l}\text { AG. Have an infection in the genital } \\
\text { area }\end{array}$ & AG. Social class & AG. Menopause End age? \\
\hline AH. Marital status? & AH. Age & AH. Education \\
\hline AI. Take adequate fruit & AI. Height? & AI. Social class \\
\hline AJ. First sex age? & AJ. Cancer vaccine taken? & AJ. Cancer vaccine taken? \\
\hline AK. Number of sex partners? & AK. Family members & AK. Height? \\
\hline AL. Menopause end age? & AL. First sex age? & AL. Family members \\
\hline AM. Never pregnant? & AM. Marital status? & AM. Never pregnant? \\
\hline AN. Cancer vaccine taken? & AN. Never pregnant? & AN. First sex age? \\
\hline AO. Problem during pregnancy? & AO. Smoker? & AO. Obese? \\
\hline AP. Food contains high fat? & AP. Obese? & AP. Smoker? \\
\hline AQ. Any birth control pill? & AQ. Source of knowledge? & AQ. Source of knowledge? \\
\hline AR. Regular exercise? & AR. How many children? & AR. How many children? \\
\hline AS. Abortion? & AS. Take alcohol? & AS. Take alcohol? \\
\hline AT. Age & AT. Number of sex partners? & AT. Number of sex partners? \\
\hline
\end{tabular}

Table 4. Data table on the significance of factors with the help of feature selection.

Info Gain Attribute Eval

A. Problem during pregnancy?

B. Abortion?

C. Have infection in genital area

D. Menopause

E. Affected by cervical cancer?

F. Affected by breast cancer

G. Knowledge about ovarian cancer?

H. Take a tentative drug?

I. Close relative affected by breast cancer
OneR Attribute Eval

A. Problem during pregnancy?

B. Abortion?

C. Have infection in genital area

D. Affected by breast cancer

E. Any birth control pill?

F. Affected by cervical cancer?

G. Regular Exercise?

H. Take a tentative drug?

I. Knowledge about ovarian cancer? 
Info Gain Attribute Eval

J. Breast cancer prior to Age 40

K. Estrogen pill taking after menopause

L. Menopause after 50?

M. Ever had a hysterectomy

N. Condom/ Diaphram

O. Any birth control pill?

P. Use napkin

Q. Any replacement therapy taken

R. Pregnancy after 35

S. pap test?

T. Children after 35

U. Regular exercise?

V. Takes hormone after menopose

W. Diet maintain

X. Cancer history in the family?

Y. Take adequate fruit

Z. STI?

AA. Food contains high fat?

AB. Age

AC. Menopause end age?

AD. Oral contraception

AE. Education

AF. Age of husband

AG. BMI?

AH. Social class

AI. Height?

AJ. Family members

AK. Cancer vaccine taken?

AL. First sex age?

AM. Source of knowledge?

AN. Marital status?

AO. How many children?

AP. Never pregnant?

AQ. Smoker?

AR. Obese?

AS. Take alcohol?

AT. Number of sex partners?

OneR Attribute Eval

J. Breast cancer prior to age 40

$\mathrm{K}$. Take adequate fruit

L. Close relative affected by breast cancer

M. Ever had a hysterectomy

N. Cancer history in the family?

O. Menopose after 50?

P. Menopause

Q. Pregnancy after 35

R. Children after 35

S. Any replacement therapy taken

T. Estrogen pill taking after menopause

U. pap test?

V. Use napkin

W. Condom/ Diaphram

$\mathrm{X}$. Diet maintain

Y. Takes Hormone after menopause

Z. STI?

AA. Menopause end age?

AB. Education

AC. Food contains high fat?

AD. Oral contraception

AE. Social class

AF. Age

AG. Age of husband

AH. BMI?

AI. Height?

AJ. Family members

AK. First sex age?

AL. Cancer vaccine taken?

AM. How many children?

AN. Smoker?

AO. Marital status?

AP. Obese?

AQ. Number of sex partners?

AR. Take alcohol?

AS. Never pregnant?

AT. Source of knowledge?

Table 5. Data table on the significance of factors with the help of feature selection.

Relief Attribute Eval

A. Problem during pregnancy?

B. Have an infection in the genital area

C. Menopause end age?

D. Abortion?

E. Family members

F. Take a tentative drug?

G. Menopause

H. Knowledge about ovarian cancer?

I. Affected by breast cancer

J. Take adequate fruit

K. Pap test?

L. Affected By cervical cancer?

M. Condom/ Diaphram

N. Food contains high fat?

O. Estrogen pill taking after menopause

P. Any birth control pill?

Q. Menopose after 50?

R. Takes hormone after menopause

S. Education

T. Use napkin

U. How many children?

V. Diet maintain

W. Age of husband

$\mathrm{X}$. Close relative affected by breast cancer

Y. Oral contraception

Z. Breast cancer prior to age 40

https://biointerfaceresearch.com/
Symmetrical Uncert Attribute Eval

A.Problem during pregnancy?

B. Abortion?

C. Have infection in genital area

D. Affected by cervical cancer?

E. Knowledge about ovarian cancer?

F. Affected by breast cancer

G. Take a tentative drug?

H. Close relative affected by breast cancer

I. Estrogen pill taking after menopause

J. Breast cancer prior to age 40

K. Menopause after 50?

L. Ever had a hysterectomy

M. Condom/ Diaphram

N. Use napkin

O. Any replacement therapy taken

P. Pap test?

Q. Pregnancy after 35

R. Any birth control pill?

S. Takes hormone after menopose

T. Children after 35

U. Diet maintain

V. Menopause

W. Regular exercise?

X. Cancer History in the family?

Y. Take adequate fruit

Z. STI? 


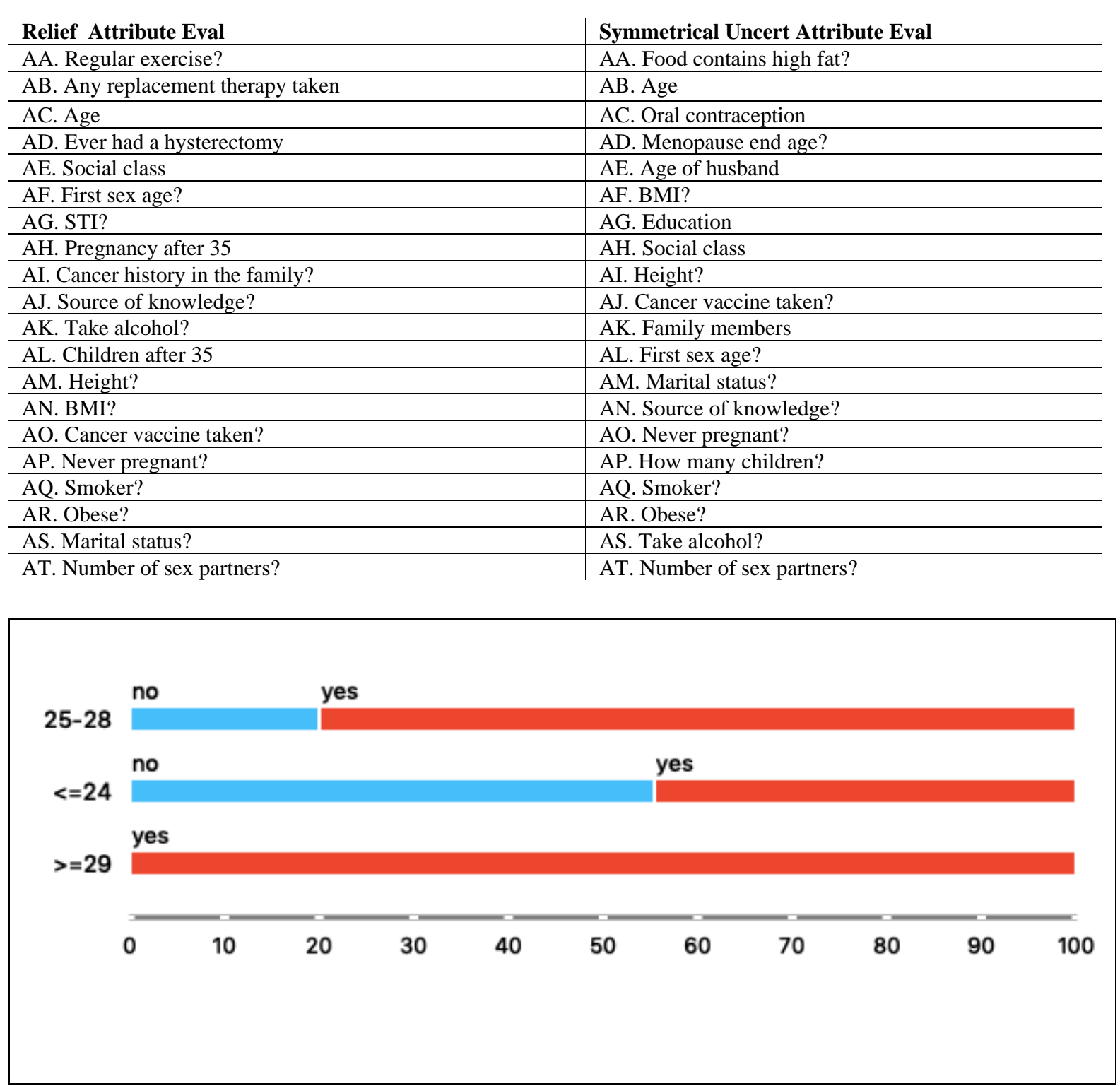

Figure 1. BMI box plot.

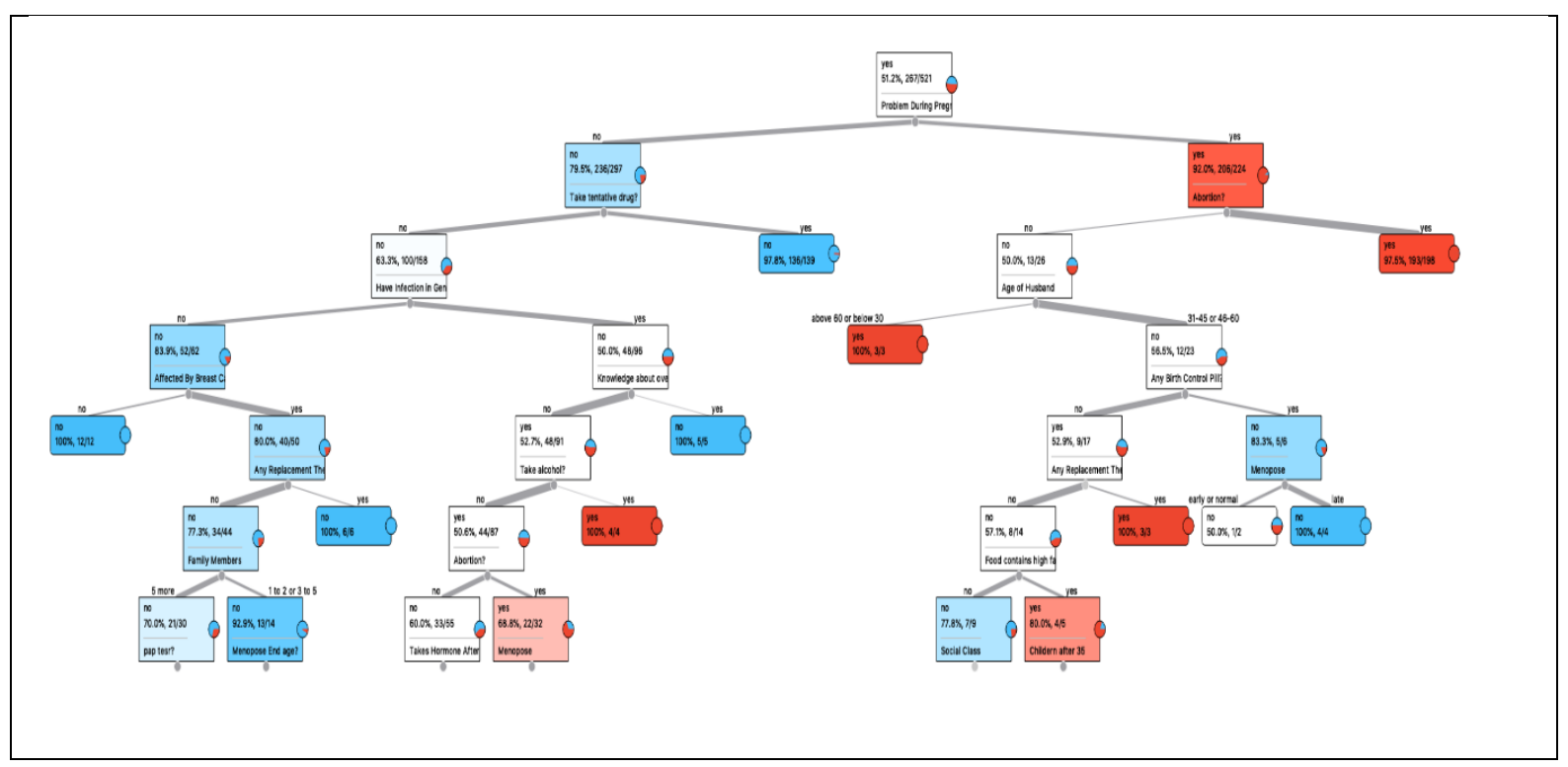

Figure 2. Decision tree among the risk factors of ovarian cancer. 


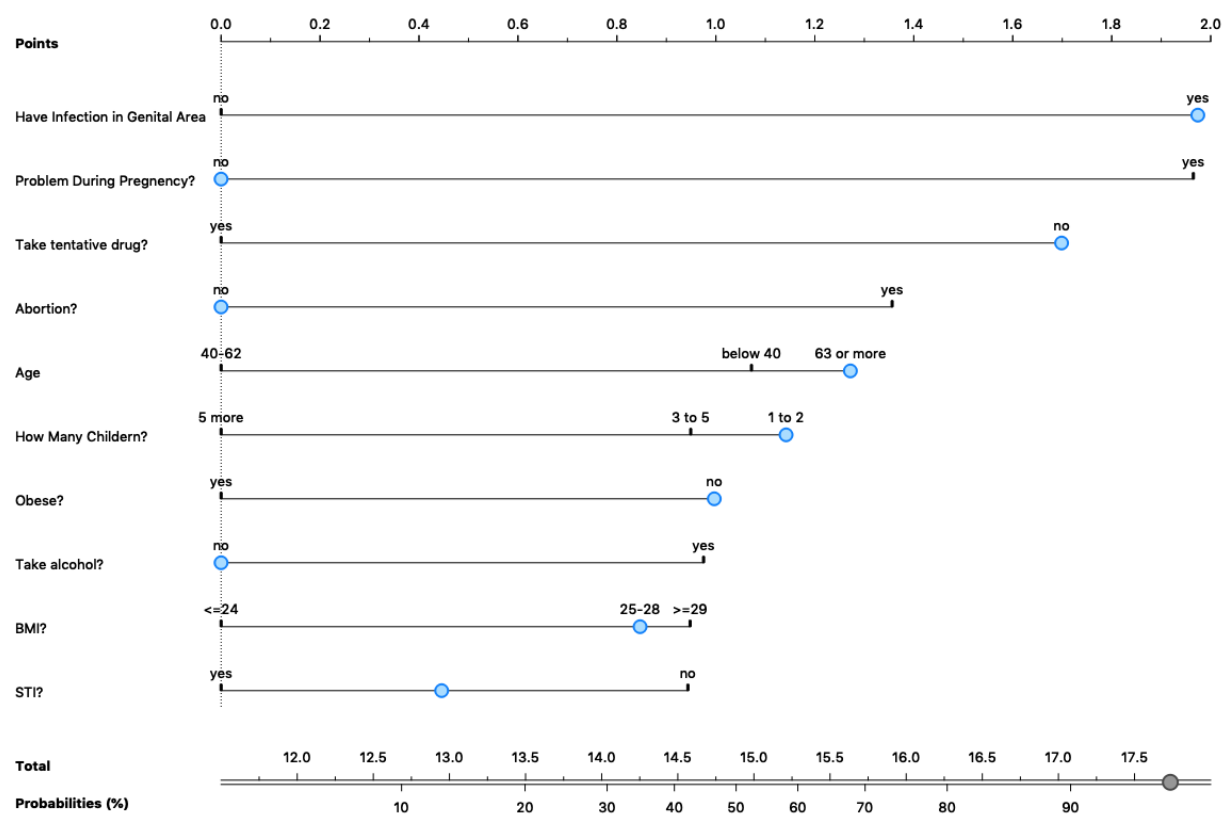

Figure 3. Probabilities distribution among the significant factors of ovarian cancer.

Figure 2 shows the decision tree of the significant risk factors of ovarian cancer where the high red portion shows the highest, light red is high, blue is medium, and white is very low risk. This tree also shows the association of the risk factors as those who have problems during pregnancy (Yes), and Abortion (Yes) has the chance of about $92 \%$ for ovarian cancer. The whole probability distribution of ovarian cancer, which shows the probability of happening ovarian cancer with the highest significant factors, has been shown in Figure 3.

\section{Conclusions}

Ovarian cancer is a woman's lifetime threat to die of 1 in 109. Total of 47 risk factors with 521 case and control group data of women were evoked by data mining and statistical and machine learning approach. About 30 are found to be linked with ovarian cancer, and 25 were considered as most significant factors by combining data mining and machine learning algorithms. The whole data set was analyzed by a machine learning model where 0.933 was the highest accuracy. The results show the significant factors along with their significance on ovarian cancer. Problem during pregnancy $\left(\chi^{2}=\mathbf{1 4 8 . 6 8 6}\right.$ pro=98.88\% $)$, infection in genital area $($ pro $=99 \%)$, abortion $\left(\chi^{2}=118\right.$ pro $\left.=\mathbf{7 4} \%\right)$, children after 35, BMI, alcohol taken , replacement therapy are extensive high significant risk factors . Husband age, age level, pregnancy after 35 are also found as highly significant factors. More data with a large no of factors should be considered in the future for more appropriate research.

\section{Funding}

This research received no external funding.

\section{Acknowledgments}

The authors are thankful to those who have took part in this research work. 


\section{Conflicts of Interest}

\section{The authors declare no conflict of interest.}

\section{References}

1. Ahmed, N.; Kadife, E.; Raza, A.; Short, M.; Jubinsky, P.T.; Kannourakis, G. Ovarian Cancer, Cancer Stem Cells and Current Treatment Strategies: A Potential Role of Magmas in the Current Treatment Methods. Cells 2020, 9, https://doi.org/10.3390/cells9030719.

2. Pirim, D.; Kaya, N.; Yıldırım, E.U.; Sag, S.O.; Temel, S.G. Characterization and in silico analyses of the BRCA1/2 variants identified in individuals with personal and/or family history of BRCA-related cancers. International Journal of Biological Macromolecules 2020, 162, 1166-1177, https://doi.org/10.1016/j.ijbiomac.2020.06.222.

3. G Guleria, S.; Jensen, A.; Toender, A.; Kjaer, S.K. Risk of epithelial ovarian cancer among women with benign ovarian tumors: a follow-up study. Cancer Causes \& Control 2020, 31, 25-31, https://doi.org/10.1007/s10552-019-01245-4.

4. Fortner, R.T.; Terry, K.L.; Bender, N.; Brenner, N.; Hufnagel, K.; Butt, J.; Waterboer, T.; Tworoger, S.S. Sexually transmitted infections and risk of epithelial ovarian cancer: results from the Nurses' Health Studies. British Journal of Cancer 2019, 120, 855-860, https://doi.org/10.1038/s41416-019-0422-9.

5. Jonsson, S.; Lundin, E.; Elgh, F.; Ottander, U.; Idahl, A. Chlamydia trachomatis and Anti-MUC1 Serology and Subsequent Risk of High-Grade Serous Ovarian Cancer: A Population-Based Case-Control Study in Northern Sweden. Translational Oncology 2020, 13, 86-91, https://doi.org/10.1016/j.tranon.2019.09.007.

6. Arul, S.; Rajagopalan, H.; Ravi, J.; Dayalan, H. Beta-Caryophyllene Suppresses Ovarian Cancer Proliferation by Inducing Cell Cycle Arrest and Apoptosis. Anti-cancer Agents Med Chem 2020, https://doi.org/10.2174/1871520620666200227093216.

7. Ray-Coquard, I.; Cibula, D.; Mirza, M.R.; Reuss, A.; Ricci, C.; Colombo, N.; Koch, H.; Goffin, F.; González-Martin, A.; Ottevanger, P.B.; Baumann, K.; Bjørge, L.; Lesoin, A.; Burges, A.; Rosenberg, P.; Gropp-Meier, M.; Harrela, M.; Harter, P.; Frenel, J.-S.; Minarik, T.; Pisano, C.; Hasenburg, A.; Merger, M.; du Bois, A.; on behalf of the, A.G.O.S.G.-1.G.E.I.C. Final results from GCIG/ENGOT/AGO-OVAR 12, a randomised placebo-controlled phase III trial of nintedanib combined with chemotherapy for newly diagnosed advanced ovarian cancer. International Journal of Cancer 2020, 146, 439-448, https://doi.org/10.1002/ijc.32606.

8. Durrani, S.; Heena, H. Controversies Regarding Ovarian Suppression and Infertility in Early Stage Breast Cancer. Cancer management and research 2020, 12, https://doi.org/10.2147/CMAR.S231524.

9. Neighbors, J.; Chase, D.; Harrow, B.; Perhanidis, J.; Monk, B.J. Gastrointestinal Symptoms and Diagnosis Preceding Ovarian Cancer Diagnosis: Delays in Diagnosis and Resulting Effects on Treatment Allocation. Gynecologic Oncology 2020, 156, e25-e26, https://doi.org/10.1016/j.ygyno.2019.11.084.

10. Natoli, M.; Bonito, N.; Robinson, J.D.; Ghaem-Maghami, S.; Mao, Y. Human ovarian cancer intrinsic mechanisms regulate lymphocyte activation in response to immune checkpoint blockade. Cancer Immunology, Immunotherapy 2020, 69, 1391-1401, https://doi.org/10.1007/s00262-020-02544-5.

11. Forstner, R. Early detection of ovarian cancer. European Radiology 2020, https://doi.org/10.1007/s00330020-06937-z.

12. Hannibal, C.G.; Frederiksen, K.; Vang, R.; Kurman, R.J.; Kjaer, S.K. Risk of specific types of ovarian cancer after borderline ovarian tumors in Denmark: A nationwide study. International Journal of Cancer 2020, 147 , 990-995, https://doi.org/10.1002/ijc.32864.

13. Udomsinkul, P.; Triratanachart, S.; Oranratanaphan, S. Risk factors for endometriotic-cyst associated ovarian cancer: A case controlled study. Taiwanese Journal of Obstetrics and Gynecology 2020, 59, 269274, https://doi.org/10.1016/j.tjog.2020.01.016.

14. Flaum, N.; Crosbie, E.J.; Edmondson, R.J.; Smith, M.J.; Evans, D.G. Epithelial ovarian cancer risk: A review of the current genetic landscape. Clinical Genetics 2020, 97, 54-63, https://doi.org/10.1111/cge.13566.

15. Su, X.; Yuan, T.; Wang, Z.; Song, K.; Li, R.; Yuan, C.; Kong, B. Two-Dimensional Light Scattering Anisotropy Cytometry for Label-Free Classification of Ovarian Cancer Cells via Machine Learning. Cytometry Part A 2020, 97, 24-30, https://doi.org/10.1002/cyto.a.23865.

16. Chen, X.; Zhang, R.; Fung, K.M.; Liu, H.; Zheng, B.; Qiu, Y. Utilizing a transfer model to classify epithelium and stroma on digital histopathological images for ovarian cancer patients. In Biophotonics and Immune Responses XV. International Society for Optics and Photonics 2020, 11241, 112410F, https://doi.org/10.1117/12.2547512.

17. Rana, H.K.; Akhtar, M.R.; Islam, M.B.; Ahmed, M.B.; Lió, P.; Huq, F.; Quinn, J.M.W.; Moni, M.A. Machine Learning and Bioinformatics Models to Identify Pathways that Mediate Influences of Welding Fumes on Cancer Progression. Scientific Reports 2020, 10, 1-15, https://doi.org/10.1016/j.jbi.2019.103313.

18. Lu, M.; Fan, Z.; Xu, B.; Chen, L.; Zheng, X.; Li, J.; Znati, T.; Mi, Q.; Jiang, J. Using machine learning to predict ovarian cancer. International Journal of Medical Informatics 2020, 141, https://doi.org/10.1016/j.ijmedinf.2020.104195. 
19. Kilicarslan, S.; Adem, K.; Celik, M. Diagnosis and classification of cancer using hybrid model based on ReliefF and convolutional neural network. Medical Hypotheses 2020, 137, https://doi.org/10.1016/j.mehy.2020.109577.

20. Huang, T.; Townsend, M.K.; Wentzensen, N.; Trabert, B.; White, E.; Arslan, A.A.; Weiderpass, E.; Buring, J.E.; Clendenen, T.V.; Giles, G.G.; Lee, I.M.; Milne, R.L.; Onland-Moret, N.C.; Peters, U.; Sandler, D.P.; Schouten, L.J.; van den Brandt, P.A.; Wolk, A.; Zeleniuch-Jacquotte, A.; Tworoger, S.S. Reproductive and Hormonal Factors and Risk of Ovarian Cancer by Tumor Dominance: Results from the Ovarian Cancer Cohort Consortium (OC3). Cancer Epidemiology Biomarkers \&amp; amp; Prevention 2020, 29, 200-207, https://doi.org/10.1158/1055-9965.EPI-19-0734.

21. Asaduzzaman, S.; Masud, F.A.; Bhuiyan, T.; Ahmed, K.; Paul, B.K.; Rahman, S.A.M.M. Dataset on significant risk factors for Type 1 Diabetes: A Bangladeshi perspective. Data in Brief 2018, 21, 700-708, https://doi.org/10.1016/j.dib.2018.10.018. 